\title{
Crucial role of noncoding RNA in driving prostate cancer development and progression
}

\author{
"Genomic regions without protein-coding potential give \\ rise to a large number of long noncoding RNA, which \\ represent close to three-times the number of protein-coding \\ genes. Studies over the past 10 years have shown that long \\ noncoding RNAs are implicated in a range of developmental \\ processes and diseases."
}

First draft submitted: 4 October 2016; Accepted for publication: 7 October 2016; Published online: 25 November 2016

Keywords: CRISPR • noncoding RNA • prostate cancer $\bullet$ risk SNP • transcription

Genomic regions without protein-coding potential give rise to a large number of long noncoding RNAs (lncRNAs), which represent close to three-times the number of protein-coding genes [1]. Studies over the past 10 years have shown that lncRNAs are implicated in a range of developmental processes and diseases [2,3]. More recently, lncRNAs have been found to be functional and clinically relevant in prostate cancer (PCa). Specifically, lncRNA PCA3 has been approved to aid PCa diagnosis by the US FDA [4], while IncRNA SChLAP1 has been shown to be an independent biomarker for metastatic PCa [5].

In general, the functional aspects of lncRNAs have been summarized as decoys, guides, scaffolds and enhancer RNAs [2,3]. Similar as well as unique mechanisms have been discovered in PCa. In castration-resistant prostate cancer, lncRNA HOTAIR has been found to interact with $A R$ and block ubiquitination of $A R$ by the E3 ubiquitin ligase, MDM2 [6]. IncRNA SChLAP1 drives PCa metastasis by binding to SWI/SNF chromatin-modifying complex and antagonizing SWI/SNF genome localization and target gene regulation [7]. IncRNA PCA3 was recently found to form a dsRNA complex with PRUNE2, a tumor suppressor gene, thereby promoting $A D A R$-mediated RNA editing [8]. Finally, IncRNA CTBP1-
AS directly suppresses CTBP1 transcription by guiding the PSF-HDAC-Sin $3 A$ complex to the CTBP1 promoter, which abolishes the androgen-mediated gene repression program [9]. Altogether, these studies have revealed the dynamic interplay between lncRNAs and cancer development, which has highlighted the importance of lncRNAs in the etiology of PCa.

Transcriptome analyses have identified hundreds of novel lncRNA transcripts that are dysregulated in PCa. IncRNAs PCAT1 and SChLAP1 were first discovered in a cohort of 102 PCa tissues and cell lines by RNA sequencing, and reported to participate in PCa tumorigenesis and progression. SChLAP1 was recently shown to have significant prognostic value for metastatic progression of PCa [5]. In addition, another lncRNA named PCAT14 was identified as a novel $\mathrm{PCa}$ - and lineage-specific lncRNA by analyzing The Cancer Genome Atlas PCa RNA-seq data, and was reported to be inversely related with disease aggressiveness [10]. However, dysregulation of lncRNAs expression does not necessarily speak for functional importance in PCa pathogenesis. Nevertheless, with rich genome, epigenome as well as transcriptome data of PCa available, it is now feasible to systematically evaluate the function of lncRNAs in PCa.
Haiyang Guo

Princess Margaret Cancer Center, University Health Network, Toronto, Ontario, Canada

Musaddeque Ahmed ${ }^{\ddagger}$ Princess Margaret Cancer Center, University Health Network, Toronto, Ontario, Canada

\section{Junjie Hua}

Princess Margaret Cancer Center, University Health Network, Toronto, Ontario, Canada

and

Department of Medical Biophysics, University of Toronto, Toronto, Ontario, Canada

\section{Fraser Soares}

Princess Margaret Cancer Center, University Health Network, Toronto, Ontario, Canada

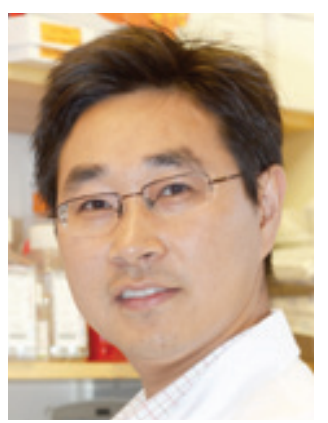

Housheng Hansen $\mathrm{He}$ Author for correspondence: Princess Margaret Cancer Center, University Health Network, Toronto, Ontario, Canada

and

Department of Medical Biophysics, University of Toronto, Toronto, Ontario, Canada

Tel.: +1 4165817736

hansenhe@uhnresearch.ca

${ }^{\ddagger}$ Authors contributed equally and are co-first authors

\section{Future $\because \%$ Medicine part of}


While PCa genome-wide association studies (GWAS) have identified multiple common risk-associated SNPs that can cumulatively explain 33\% of familial risk [11], very little is known about the effect of risk-associated SNPs on lncRNAs [12]. To systematically evaluate lncRNAs involved in PCa pathogenesis, we performed an integrative analysis of the lncRNA transcriptome with epigenomic and risk SNP data [13]. Interestingly, we found that PCa-risk SNPs are significantly enriched in the regulatory regions marked by DNase I hypersensitive sites (DHSs) but not in the exons of proteincoding genes or lncRNAs. Notably, we observed that PCa-risk SNPs were specifically enriched in the cistromes of $\mathrm{PCa}$-related transcription factors, such as $A R$ and HOXB13. Upon further analysis, these risk-SNP containing DHSs were in close proximity to lncRNAs, suggesting a potential cross talk between risk SNP and lncRNA regulation. Integrative analysis of SNPs in the regulatory regions identified 45 candidate lncRNAs associated with $50 \%$ of the PCa risk loci.

The top hit by our prediction was lncRNA PCAT1, which is specifically expressed in prostate tissues and $\mathrm{PCa}$, and promotes PCa cell proliferation [14]. Consistent with previous reports, we found that PCAT1 is significantly upregulated in primary $\mathrm{PCa}$ compared with benign prostate tissues, and knockdown of PCAT1 expression suppressed $\mathrm{PCa}$ cell proliferation and tumor growth.

\section{"To systematically evaluate IncRNAs involved in PCa pathogenesis, we performed an integrative analysis of the IncRNA transcriptome with epigenomic and risk SNP data."}

PCAT1 is located in the $8 \mathrm{q} 24$ gene desert region, which contains $10 \mathrm{PCa}$ risk loci. Interestingly, a PCaspecific DHS harboring the risk SNP rs7463708 was predicted to interact with $P C A T 1$ promoter. By performing chromosome conformation capture (3C), we were able to validate this interaction. Importantly, the PCAT1 promoter--enhancer interaction was specific to risk ' $T$ ' allele of rs 7463708 . The rs 7463708 locus with risk allele also showed higher enhancer activity than nonrisk allele, and disruption of rs 7463708 locus resulted in the decrease of PCAT1 expression. In further analyses, we identified that the risk allele 'T' of rs 7463708 created a stronger ONECUT2 motif at PCAT1 enhancer, resulting in allele-specific occupancy of transcription factors ONECUT2 and $A R$. In addition, chromatin immunoprecipitation experiments revealed that the recruitment of $A R$ at the PCAT1 enhancer was dependent on ONECUT2 occupancy. In agreement with our binding data, the induction of PCAT1 by prolonged androgen treatment was dependent on ONECUT2 expression. Finally, we demon- strated that $P C A T 1$ interacts with $A R$ and $L S D 1$, which mediates their occupancy at the enhancers of GNMT and DHCR24, two androgen late-response genes that participate in PCa development and progression. Altogether, these data establish the capacity of risk SNPs to promote PCa development by modulating lncRNA expression, and provide additional evidence that lncRNAs, such as PCAT1 are crucial to PCa.

The catalog of IncRNA transcripts in cancer has grown tremendously during the past few years, but the functions of most lncRNAs are still unclear. Our integrative analysis in PCa retrieves the intrinsic connections between risk SNPs and functional lncRNAs from the genome, epigenome and transcriptome data. Since these data are becoming increasingly available for many cancer types, our pipeline will also be useful for the lncRNA study in other cancer types.

Many GWAS SNPs also locate in lncRNA gene bodies [1]. Since most lncRNAs function through interaction with other molecules (such as RNA, DNA and protein), some studies have revealed that those interactions have a sequence-specific pattern [15]. However, it remains to be examined whether these GWAS SNPs located in lncRNAs mediate functional interactions. In addition, with newer approaches being recently established to analyze lncRNA interactomes [16,17], it is time to investigate the implication of SNPs in the lncRNA interactomes.

Recently, two studies reported somatic mutations enriched in regulatory regions that were linked to promoter and nucleotide excision repair activities $[18,19]$. These somatic mutations in regulatory regions may also modulate lncRNA expression and thus affect tumor growth and progression. Although there are differences in downstream mechanistic analyses of genetic predisposition and acquired variation data, with appropriate modifications, our computational and experimental workflow could be applied for identifying lncRNAs affected by acquired variants in cancer.

Genome editing technologies have been used to validate the function of genetic variations. Transcription activator-like effector nuclease or CRISPR-Cas9 induced homology-directed repair can introduce allele-specific modification to the genome, which has been proved to be a powerful tool to validate the causal SNPs in regulatory regions [20]. In addition, cell lines derived from single-cell cloning with different allelic makeup of a mutation, offer an ideal model to study the allelic dependence of transcription factor binding and subsequent effect on gene expression. To further expand the scale of functional validation of lncRNArelated SNPs, CRISPR-Cas9 based functional screens should be utilized to manipulate the activity of noncoding regions systematically. Thus, our integrative 
analysis provides a focused list of lncRNA and SNPs that are likely regulating these $\operatorname{lncRNAs}$. Robust genome editing techniques like CRISPR-Cas9 can immensely contribute to validate the causality by these SNPs and their modus operandi.

\section{Financial \& competing interests disclosure}

Work in the laboratory of $\mathrm{HH} \mathrm{He}$ is supported by Princess Margaret Cancer Foundation (to $\mathrm{HH} \mathrm{He}$ ), Canada Foundation for Innovation and Ontario Research Fund (CFI32372 to $\mathrm{HH} \mathrm{He}$ ), NSERC Discovery Grant (498706 to $\mathrm{HH} \mathrm{He}$ ), WICC Ontario 20th Anniversary Prostate Cancer Innovation Grant

\section{References}

1 Iyer MK, Niknafs YS, Malik R et al. The landscape of long noncoding RNAs in the human transcriptome. Nat. Genet. 47(3), 199-208 (2015).

2 Geisler S, Coller J. RNA in unexpected places: long noncoding RNA functions in diverse cellular contexts. Nat. Rev. Mol. Cell. Biol. 14(11), 699-712 (2013).

3 Quinn JJ, Chang HY. Unique features of long non-coding RNA biogenesis and function. Nat. Rev. Genet. 17(1), 47-62 (2016).

4 Mouraviev V, Lee B, Patel V et al. Clinical prospects of long noncoding RNAs as novel biomarkers and therapeutic targets in prostate cancer. Prostate Cancer Prostatic Dis. 19(1), 14-20 (2016).

5 Prensner JR, Zhao S, Erho N et al. RNA biomarkers associated with metastatic progression in prostate cancer: a multi-institutional high-throughput analysis of SChLAP1. Lancet Oncol. 15(13), 1469-1480 (2014).

6 Zhang A, Zhao JC, Kim J et al. LncRNA HOTAIR enhances the androgen-receptor-mediated transcriptional program and drives castration-resistant prostate cancer. Cell Rep. 13(1), 209-221 (2015).

7 Prensner JR, Iyer MK, Sahu A et al. The long noncoding RNA SChLAP1 promotes aggressive prostate cancer and antagonizes the SWI/SNF complex. Nat. Genet. 45(11), 1392-1398 (2013).

8 Salameh A, Lee AK, Cardó-Vila M et al. PRUNE2 is a human prostate cancer suppressor regulated by the intronic long noncoding RNA PCA3. Proc. Natl Acad. Sci. USA 112(27), 8403-8408 (2015).

9 Takayama K, Horie-Inoue K, Katayama $S$ et al. Androgenresponsive long noncoding RNA CTBP1-AS promotes prostate cancer. EMBO J. 32(12), 1665-1680 (2013).

10 Shukla S, Zhang X, Niknafs YS et al. Identification and validation of PCAT14 as prognostic biomarker in prostate cancer. Neoplasia 18(8), 489-499 (2016). of the CCS (703800 to HH He), CIHR Transitional Operating Grant (142246 to HH He), Movember Rising Star Award and Discovery Grant from Prostate Cancer Canada. HH He holds an OMIR Early Researcher Award and a New Investigator Salary Award from the CIHR. J Hua is a CIHR Graduate Student Fellowship recipient. The authors have no other relevant affiliations or financial involvement with any organization or entity with a financial interest in or financial conflict with the subject matter or materials discussed in the manuscript apart from those disclosed.

No writing assistance was utilized in the production of this manuscript.

11 Al Olama AA, Kote-Jarai Z, Berndt SI et al. A metaanalysis of 87,040 individuals identifies 23 new susceptibility loci for prostate cancer. Nat. Genet. 46(10), 1103-1109 (2014).

12 Hazelett DJ, Rhie SK, Gaddis M et al. Comprehensive functional annotation of 77 prostate cancer risk loci. PLoS Genet. 10 (1), e1004102 (2014).

13 Guo H, Ahmed M, Zhang F et al. Modulation of long noncoding RNAs by risk SNPs underlying genetic predispositions to prostate cancer. Nat. Genet. 48(10), 1142-1150 (2016).

14 Prensner JR, Chen W, Iyer MK et al. PCAT-1, a long noncoding RNA, regulates BRCA2 and controls homologous recombination in cancer. Cancer Res. 74(6), 1651-1660 (2014).

15 Ray D, Kazan H, Chan ET et al. Rapid and systematic analysis of the RNA recognition specificities of RNA-binding proteins. Nat. Biotechnol. 27(7), 667-670 (2009).

16 Nguyen TC, Cao X, Yu P et al. Mapping RNA-RNA interactome and RNA structure in vivo by MARIO. Nat. Commun. 7, 12023 (2016).

17 Lu Z, Zhang QC, Lee B et al. RNA Duplex map in living cells reveals higher-order transcriptome structure. Cell 165(5), 1267-1279 (2016)

18 Sabarinathan R, Mularoni L, Deu-Pons J, Gonzalez-Perez A, Lopez-Bigas N. Nucleotide excision repair is impaired by binding of transcription factors to DNA. Nature 532(7598), 264-267 (2016).

19 Perera D, Poulos RC, Shah A, Beck D, Pimanda JE, Wong JW. Differential DNA repair underlies mutation hotspots at active promoters in cancer genomes. Nature 532(7598), 259-263 (2016).

20 Spisak S, Lawrenson K, Fu Y et al. CAUSEL: an epigenomeand genome-editing pipeline for establishing function of noncoding GWAS variants. Nat. Med. 21(11), 1357-1363 (2015). 